\title{
Using Second-derivative Filters to Assist in Width Estimations of Size Exclusion Chromatography Signal Peaks with Static Light-scattering Detections to Obtain More Accurate Molecular Weight
}

\author{
Po-Ting Chen, Hsiao-Ping Chen, Chia-Hung Hung, and Shau-Chun WAng ${ }^{\dagger}$ \\ Department of Chemistry and Biochemistry and Center for Nano Bio-detection Technology, AIM-HI, National \\ Chung Cheng University, Chia-Yi 621, Taiwan
}

\begin{abstract}
Static light-scattering (LS) detection can determine the molecular weight $\left(M_{\mathrm{W}}\right)$ of polymers eluted with size-exclusion chromatography (SEC) without using any standards when the differential refraction index (RI) of solutes are obtained. On the other hand, the noisy chromatographic signal peak acquired using a static LS detector often causes difficulty in peak-width recognition. This disadvantage limits the determination accuracy and precision of the $M_{\mathrm{W}}$ values. This study developed one second-order derivative filtering procedure by convolving the original LS chromatogram against the second-derivative curve of one artificial Gaussian-shape chromatographic peak to suppress the noises and to correct the baseline of the chromatogram. More accurate estimations of the chromatographic peak widths of pullulan samples were achieved to improve the $M_{\mathrm{W}}$ determination accuracy. For noisy original chromatography peaks of pullulan $5 \mathrm{k}$ (SNR of approximately 10 ), the non-ideal determination accuracy of the $M_{\mathrm{W}}$ values $(9.3 \%)$ is improved to $-1.3 \%$ with the assistance of the filtering procedures.
\end{abstract}

Keywords Static light-scattering, size-exclusion chromatography, second-derivative filter

(Received May 16, 2014; Accepted October 3, 2014; Published November 10, 2014)

\section{Introduction}

During the past 50 years, size-exclusion chromatography (SEC) has been the most preeminent tool among polymer characterization techniques. Typically, an SEC column packed with porous beads separates based on the polymer-analyte size, such as the hydrodynamic volume. Such a separation tool can determine the polymer molecular weight $\left(M_{\mathrm{W}}\right)$ values, even if these values are not absolute, and are only relative to a particular calibration standard. ${ }^{1}$

Scattering light from polymer solutes is detected with a static light-scattering (LS) detector. When the solute molecules are considered to be Rayleigh scatters in theory, the scattering light intensity can determine the $M_{\mathrm{W}}$ of a polymer.

The approximate form of the Zimm equation describes the quantitative relation between the scattering intensity, $R$, and the polymer $M_{\mathrm{W}}$ (less than $100 \mathrm{k} \mathrm{Da}$ ) in a diluted solution, as follows:

$$
\frac{K c}{R(c, q)} \cong \frac{1}{M_{\mathrm{W}}}
$$

where $c$ is the polymer concentration, $q=\left(4 \pi \mathrm{n}_{0} / \lambda\right) \sin (\theta / 2), \mathrm{n}_{0}$ is the solvent refraction index (RI), and $\lambda$ and $\theta$ are the wavelength of incident light and the collected angle of scattering

$\dagger$ To whom correspondence should be addressed.

E-mail: chescw@ccu.edu.tw light, respectively. In Eq. (1), $K$ is an equipment constant determined by the differential refraction index of solutes $(\mathrm{dn} / \mathrm{dc})$, where $\mathrm{n}$ is solution RI. ${ }^{2}$

When one polymer analyte is eluted from an SEC column, of which the chromatographic peaks are acquired with two detectors in tandem, including one mass sensitive detector (e.g., RI detector) proportionally responding to the solute concentration, and the other static LS detector, the inverse of $M_{\mathrm{W}}$, can be obtained by the ratio of $K c / R$ in a dilute solution.

Therefore, the RI detector and a static LS detector are often used in tandem to couple with the SEC column as one of the primary detection tools to characterize the polymer $M_{\mathrm{W}}$, especially for a low $M_{\mathrm{W}}$, without using any polymer standards. When the dimension of the polymer analyte is large and the $M_{\mathrm{W}}$ is greater than $100 \mathrm{k} \mathrm{Da}$, low-angle detection of scattering light is used to maintain a proportionally valid unity. Alternatively, other size-sensitive detections, such as differential viscometry, are used with RI and LS detections to correct the determinated deviation. ${ }^{3}$

Limited by the typical sub-micron size of polymers, the scattering light intensity of an SEC-eluted analyte is easily buried in noise. The chromatograms acquired using static LS detectors are often noisy, especially when solutes are at a low concentration, which causes difficulty in recognizing the chromatographic peak range. Because the $M_{\mathrm{W}}$ values are estimated as averaged values of polymer eluted within the same peak, unreliable peak range estimations occasionally result in the determinations of $M_{\mathrm{W}}$ values with unacceptable errors. ${ }^{4}$

One mass-sensitive detector, such as an RI detector, is often 
used with a static LS detector in tandem to couple with the SEC column to facilitate average $M_{\mathrm{W}}$ determinations. Although the chromatograms acquired using the RI detector are usually less noisy than those acquired using a static LS detector, because of its superior sensitivity, the visual width range of the chromatographic peaks of these two types of detectors are not the same, because RI is mass sensitive, whereas LS is size sensitive.

Recent publications have pointed out the usefulness of using the LS detector with SEC as a process analysis tool to monitor the $M_{\mathrm{W}}$ 's variability of manufactured polymers..$^{5-7}$ Therefore, there remains a need to develop analytical techniques to correct the estimated $M_{\mathrm{W}}$ results to improve the determination accuracy.

The spectroscopy signal-processing technique with second-derivative filters ${ }^{8,9}$ uses the reference signal, typically the second-derivative curve of one Gaussian profile, to convolve against the detected signals for processing, and including chromatograms, have been used to correct the drifted baselines of chromatograms and to increase the signal-to-noise ratios (SNRs) of the chromatographic peaks. ${ }^{10,11}$ In addition to improving the signal quality, second-derivative filtering results in a flat baseline of zero background in the convolved chromatogram. Therefore, the edges of the convolved signal peak cross the corrected baseline at the zero level, and are easier to locate when the interested peak is separated from the nearest peaks.

In our previous study, ${ }^{11}$ the algorithm used to perform second-derivative filtering to process a digitized chromatogram exhibited the following relation. When the M-th data point in the original chromatogram convolved with the $\mathrm{N}$-th data point in the second-derivative profile, the projected point was the $\mathrm{M}+\mathrm{N}-1$ point in the final convolved chromatogram. Because the edge point in the convolved chromatogram is projected from the convolution of the edge of the original chromatographic peak and the edge of the second-derivative reference profile, the location of the edge of the original chromatographic peak $\mathrm{M}$ can be found when the edge of the convolved signal is located at the $\mathrm{M}+\mathrm{N}-1$ point with the edge of the reference profile specified at the $\mathrm{N}$-th point.

In this study, the apex point of the chromatographic peak acquired using an RI detector was used as the center point of the reference Gaussian shape signal. The half-height width of the RI peak is equal to the 2.35-fold standard deviation of this Gaussian peak. The edges of the Gaussian profile (the N-th point) were designated as the points at a distance of three-fold standard deviation away from the center point. Using the second-derivative profile of this Gaussian peak to convolve against the original LS chromatogram, the edges of the convolved peak crossing the baseline near the zero level were easy to find manually. The $\mathrm{M}$-th point in the original chromatogram was projected to the $\mathrm{M}+\mathrm{N}-1$ point when it convolved with the $\mathrm{N}$-th point in the reference profile. Using this relation, the edges of the noisy peak in the original LS chromatogram can be located to determine the peak width for estimating the $M_{\mathrm{W}}$ values of polymer samples.

Figure 1a shows one well-separated case, in which the resolution of two peaks in the original simulated chromatogram in the upper frame is 2.2. The convolved trace of these two peaks in the lower frame are both similar to the second-derivative curve of one Gaussian peak, and either edge point of each peak are projected to the positions close to the baseline. For instance, the edge points of the larger peak, marked as A and B in the original trace, are convolved to project to the points $\mathrm{C}$ and $\mathrm{D}$ near the baseline in the filtered trace. In Fig. 1b when two peaks in the original simulated chromatogram in the upper frame, of which the resolution is only 1.5 , are not ideally separated, although the convolved trace of the larger peak in the lower frame still contains the features of one second-derivative Gaussian peak, the right edge of the convolved trace does not reach the baseline. In this case, the turning point, $\mathrm{D}^{\prime}$, between two convolved peak traces can be used as the projection point of the peak right edge at point $\mathrm{B}^{\prime}$. The left edge point of the higher peak at point $\mathrm{A}^{\prime}$ projected to point $\mathrm{C}^{\prime}$ approaching the baseline of the convolved trace is also easy to locate. As the separation resolution becomes even more deteriorated, Fig. 1c shows the convolved trace when two peaks in the original simulated chromatogram of which the resolution is only 1.3. Although the convolved trace of the larger peak still contains the features of one second-derivative Gaussian peak, the right edge of the trace is difficult to find one turning adjacent in the filtered chromatogram. Therefore, using the second-derivative filter method to determine the more accurate peak width would become not useful when the separation resolution is 1.3 or less.

Because it is easier to locate the edges in the convolved chromatographic peak when the separation resolution is greater than 1.3 , the determinations of the $M_{\mathrm{W}}$ values are more accurate than the estimations using the LS peak width. In this paper, to prove the $M_{\mathrm{w}}$ 's determination accuracy improvement, the mixture of pullulan $400 \mathrm{k}$ and pullulan $5 \mathrm{k}$ is separated with adequate resolution to determine the $M_{\mathrm{W}}$ 's of these two polymers via second-derivative filtering procedures.

\section{Experimental}

\section{Reagents and chemicals}

The standards of linear polysaccharide, pullulan $400 \mathrm{k}$ and pullulan $5 \mathrm{k}$, to prepare sample solutions were obtained from American Polymer Standards Corp. (Mentor, OH). Detector calibration standards, such as polyethylene oxide (PEO $21 \mathrm{k}$, $\left.M_{\mathrm{W}} 21216\right)$ and pullulan $200 \mathrm{k}\left(M_{\mathrm{W}} 20000\right)$, were provided by Scientech (Taipei, Taiwan). The OHpak SB-806M HQ SEC column $(8.0 \mathrm{~mm} \times 30 \mathrm{~cm})$ was from Shodex (Showa Denko, K.K., Japan). Deionized water was made by a Milli-Q water purification system (Millipore, Bedford, MA) to prepare polymer solutions and SEC mobile-phase solvents.

\section{Preparations of sample and mobile-phase solutions}

Pullulan standards were dissolved in deionized water, and remained overnight to prepare stock solutions $(3 \mathrm{mg} / \mathrm{mL})$ stored at $4^{\circ} \mathrm{C}$. The stock solutions were diluted with deionized water to make up sample solutions of lower concentrations prior to use. The deionized water was filtered with a $0.45 \mu \mathrm{m}$ membrane and degassed via sonication for $30 \mathrm{~min}$ to use as mobile-phase solvent.

\section{Apparatus}

The SEC system composed of a solvent delivery module (Viscotek VE 1122) and a multiplex detection module TDA 302 with a static LS detector collecting signals at 90-degree right angle or 7-degree low angle, viscometry detector, RI detector, and UV/Vis absorbance detector, was from Malvern Instrument (UK).

\section{Procedure}

The SEC operation conditions were set as follows: injection volume, $100 \mu \mathrm{L}$; flow rate, $0.8 \mathrm{~mL} / \mathrm{min}$; and run time, $25 \mathrm{~min}$. In our experiment the temperature was maintained at $30^{\circ} \mathrm{C}$ inside a TDA 302 detection module to record chromatograms with static LS signals at right angle and RI signals simultaneously. 
(a) Original simulated chromatogram
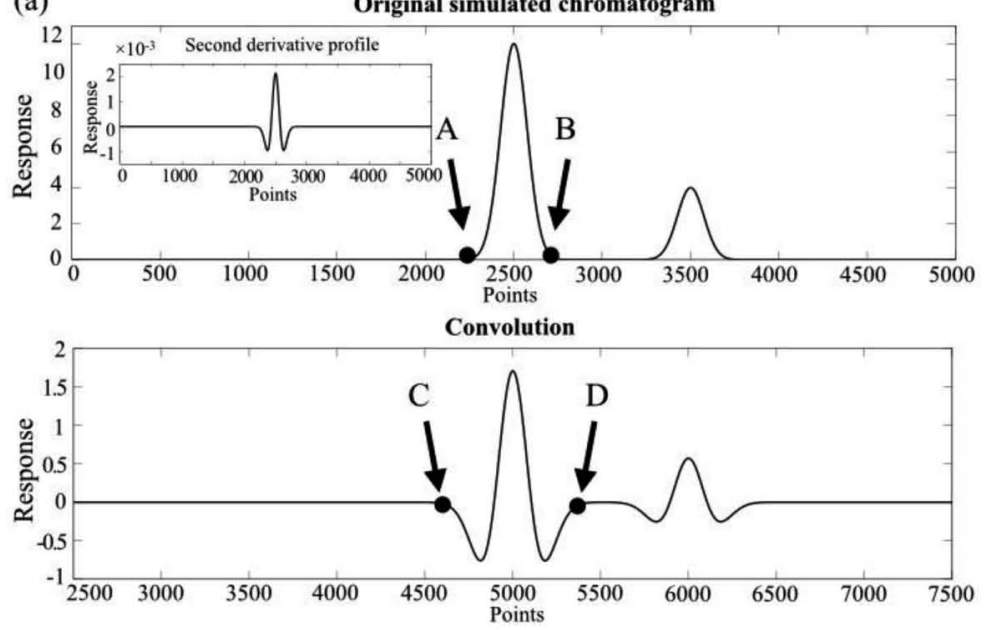

(b) Original simulated chromatogram

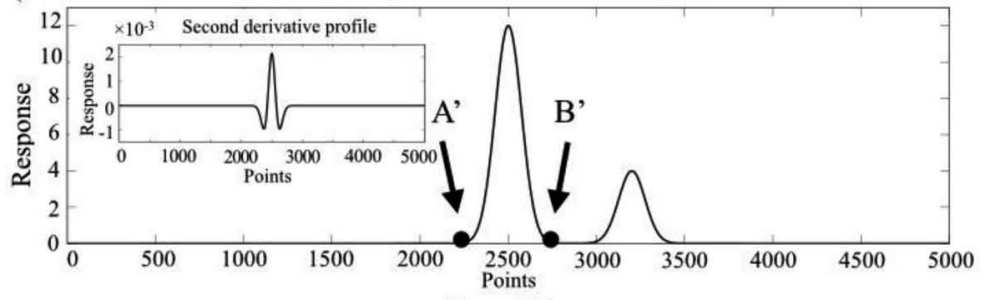

Convolution

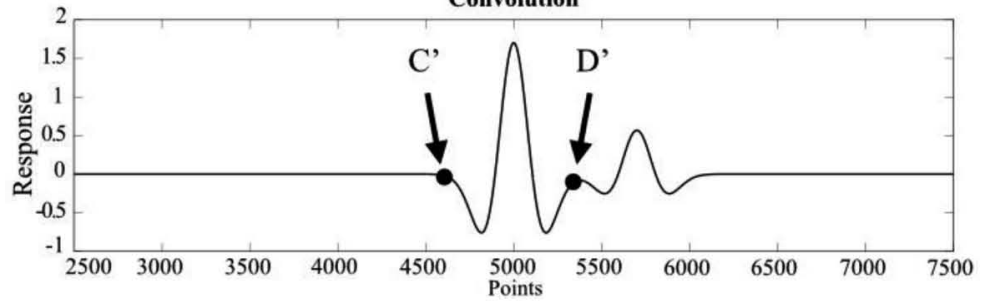

(c) Original simulated chromatogram
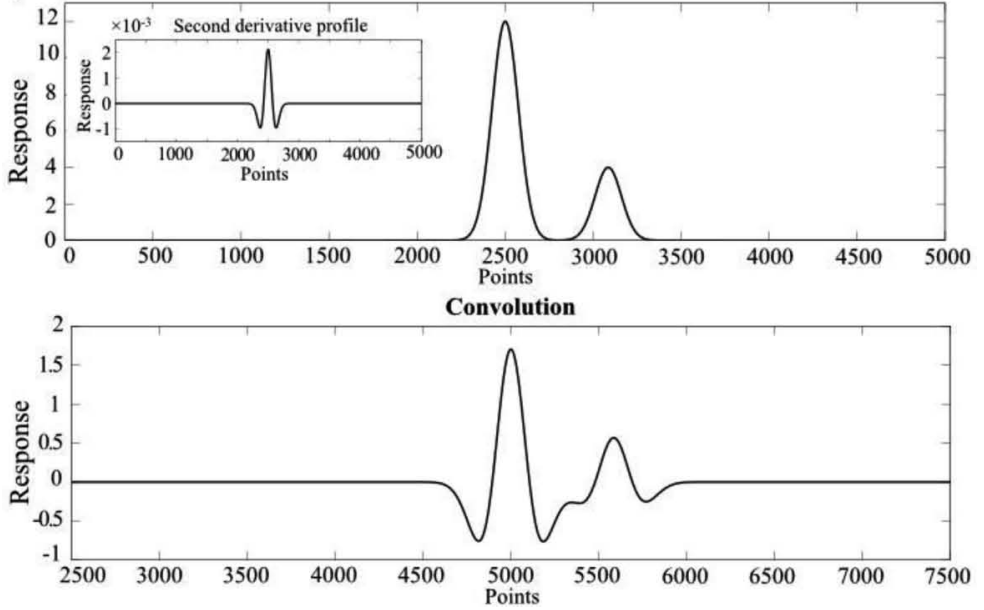

Fig. 1 The artificial chromatograms contain two peaks with various separation resolutions (upper frame) and their filtered chromatograms resulting from convolving the original chromatograms against a second-derivative Gaussian curve (lower frame). The imbeded graphs in the upper frame show the second-derivative curves of the Gaussian peak of which the center and width are the same as these parameters of the left peak in the original chromatograms. (a) Two well-separated signal peaks of which the separation resolution is 2.2. The edge points of the left peak in the original chromatogram (A and B) are projected to points $\mathrm{C}$ and $\mathrm{D}$ in the filtered frame. (b) Two non-ideally separated signal peaks shown of which the separation resolution is 1.5. The edge points of the left peak in the original chromatogram $\left(\mathrm{A}^{\prime}\right.$ and $\left.\mathrm{B}^{\prime}\right)$ are projected to points $\mathrm{C}^{\prime}$ and $\mathrm{D}^{\prime}$ in the filtered chromatogram. (c) Two inadequately separated signal peaks of which the separation resolution is only 1.3. Two overlapping peaks appear in the central part of a convolved chromatogram, of which the peak edges become no longer recognizable. 


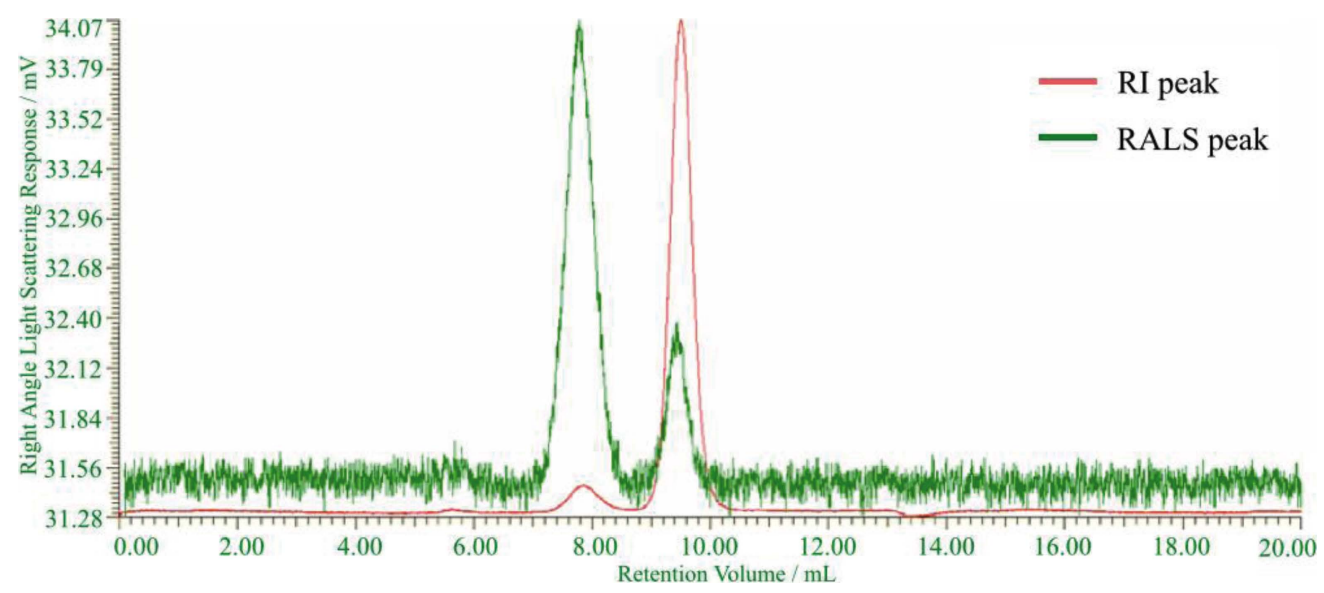

Fig. 2 Chromatograms of the sample containing pullulan $5 \mathrm{k}$ of $0.8 \mathrm{mg} / \mathrm{mL}$ and pullulan $400 \mathrm{k}$ of $0.07 \mathrm{mg} / \mathrm{mL}$ acquired with a static LS detector (the green trace) collecting right-angle scattering intensity signals and RI detector (the red trace), respectively.

A polymer standard, such as PEO $21 \mathrm{k}\left(M_{\mathrm{W}} 21216\right)$, was used to calibrate the instrument response and to verify the relation of the Rayleigh ratio versus the polymer $M_{\mathrm{W}}$ value described in Eq. (1). In addition, the pullulan $200 \mathrm{k}$ standard was injected to obtain the $\mathrm{dn} / \mathrm{dc}$ value to determine the $M_{\mathrm{W}}$ values of other pullulan samples (400 k and $5 \mathrm{k}$ ) using chromatograms acquired with right-angle LS detection and RI detection. Optionally, the chromatograms with viscometry detection were obtained to correct any determinations.

\section{Data acquisition and signal processing}

Chromatographic data were processed with the built-in application software package (OmniSEC 4.6) provided by the detector vendor to estimate the values of the polymer $M_{\mathrm{W}}$ Prior to estimating the $M_{\mathrm{W}}$ values when necessary, the data were exported to a personal computer to process with second-derivative filtering routines in the codes of MATLAB 6.1 (Mathworks, MA) previously developed in our laboratory. ${ }^{11}$

\section{Results and Discussion}

The differences of the $M_{\mathrm{W}}$ values of pullulan $400 \mathrm{k}$ and pullulan $5 \mathrm{k}$ determined with and without viscometry detection chromatograms are found to be negligible (within 5\%). Therefore, angle corrections of the $M_{\mathrm{W}}$ determination using right-angle LS detection are unnecessary in this study.

Figure 2 shows the chromatograms of samples containing pullulan $5 \mathrm{k}\left(M_{\mathrm{W}} 6438 \mathrm{Da}\right)$ of concentration $0.8 \mathrm{mg} / \mathrm{mL}$ and pullulan $400 \mathrm{k}\left(M_{\mathrm{W}} 383250 \mathrm{Da}\right)$ of $0.07 \mathrm{mg} / \mathrm{mL}$ acquired with a static LS detector collecting right-angle signals (the green trace) and an RI detector (the red trace, respectively. Although the peaks of these two analytes are adequately separated in the static LS chromatogram (the resolution is about 1.48), reliably locating the exact range of each chromatographic peak width is somewhat difficult because these signal peaks are noisy, especially to identify the width range of the pullulan $5 \mathrm{k}$ peak, of which the SNR is only about ten, riding on one somewhat shifted baseline. The accurate determinations of $M_{\mathrm{W}}$ values are therefore problematic without accurately estimating the peak width ranges.

When the peak width ranges in the original LS chromatogram are given, the $M_{\mathrm{W}}$ values of pullulan $5 \mathrm{k}$ and pullulan $400 \mathrm{k}$ are obtained using the OmniSEC software pack provided by the detector vendor. With eleven replicate sample injections the $M_{\mathrm{W}}$ calculation results showed that the mean values of the pullulan $5 \mathrm{k}$ and the pullulan $400 \mathrm{k}$ molecular weights are $7036 \mathrm{Da}$ and $386470 \mathrm{Da}$, respectively. The determination accuracy of the $M_{\mathrm{W}}$ values using pullulan $5 \mathrm{k}$ and pullulan $400 \mathrm{k}$ are 9.3 and $0.8 \%$, respectively. Apparently the molecular-weight determination of pullulan $5 \mathrm{k}$ is not accurate.

For the convenience to determine the signal locations on the convolved chromatogram, where the end points of the original chromatographic peak are projected, the segment of the original static LS chromatogram in Fig. 2 between the retention volume, 2.68 and $16.0 \mathrm{~mL}$, is re-plotted in Fig. 3a by replacing the retention volume in the $x$-axis as a sequence of data points. The imbeded graph shows the second-derivative profile of one Gaussian peak of that the center and standard deviation are estimated with the RI peaks of pullulan $5 \mathrm{k}$ in Fig. 2. (The $x$-axis of the imbeded graph has also been converted to a sequence of data points.) The points that are two folds of the standard deviation away from the center of the reference profile are found as the 2373-th and 2627-th point, respectively. Having convolved with the second-derivative trace, the original static LS chromatogram in Fig. 3a is filtered to smooth any noisy signal peaks, and to correct any shifted baseline. The central part of the filtered chromatogram via the above convolution procedures is zoomed in Fig. 3b. The smaller peak in Fig. 3b, of which the shape is similar to a second-derivative curve of a Gaussian profile, is the filtered signal of the pullulan $5 \mathrm{k}$ peak in the original chromatogram (Fig. 3a). This peak clearly shows the features of a convolved chromatographic peak with a second-derivative filter, which contain one positive peak between the other two negative peaks.

As explained in the Introduction section, because the resolution between two pullulan peaks in the original LS chromatographic peak is not large enough (1.48), the left edge of the pullulan $5 \mathrm{k}$ peak is not expected to approach the baseline in the filtered chromatogram. Therefore, toward the left-hand side along the filtered trace of pullulan $5 \mathrm{k}$, one can find one turning adjacent to the filtered trace of pullulan $400 \mathrm{k}$, as the projection point of the left edge of the pullulan $5 \mathrm{k}$ peak. Toward the right-hand side along the filtered trace of pullulan $5 \mathrm{k}$, one zero-crossing point is found as the projection point of the right edge. These two projected points of both edges are marked as C and D in the 
(a)

Original LS chromatogram

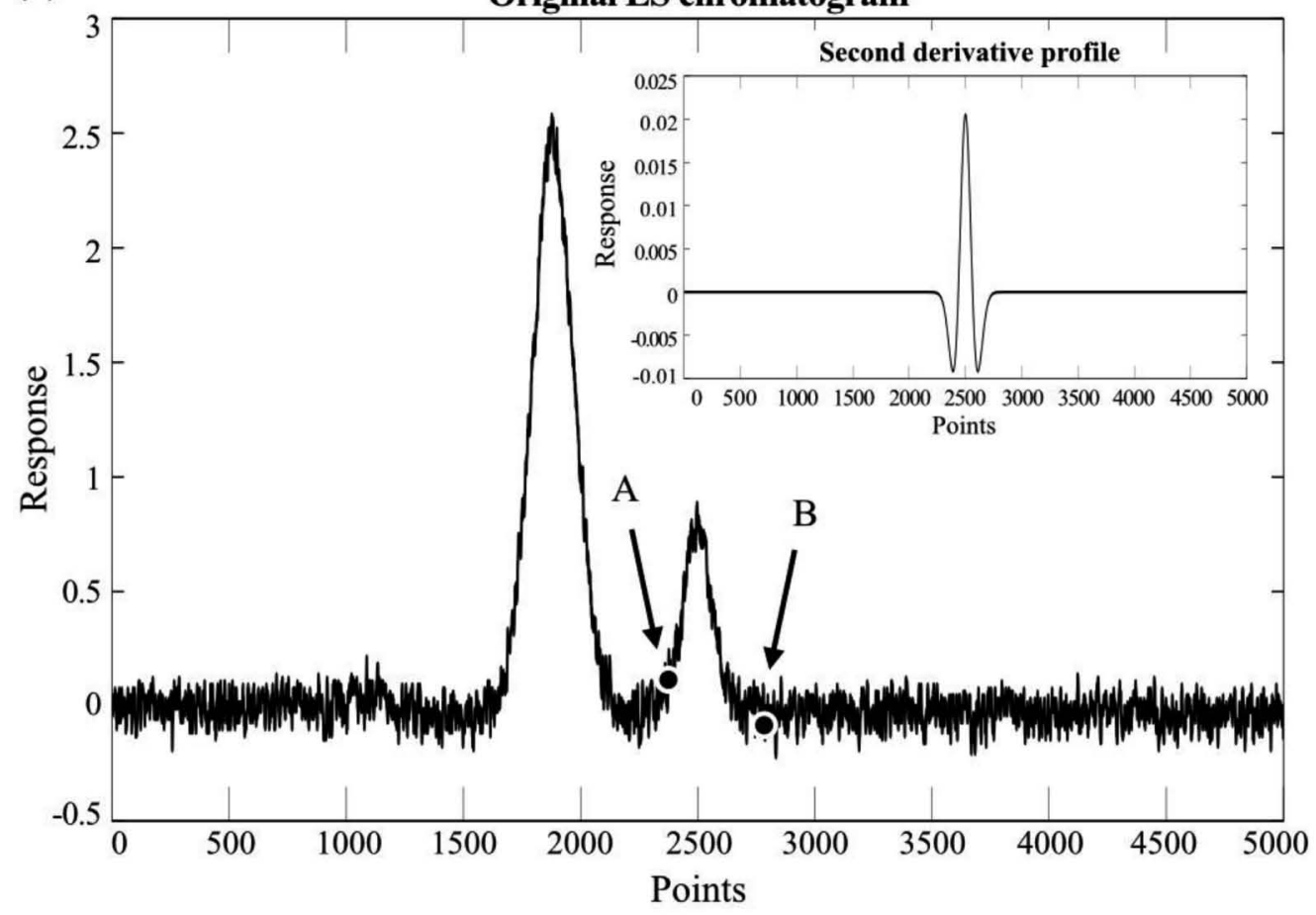

(b)

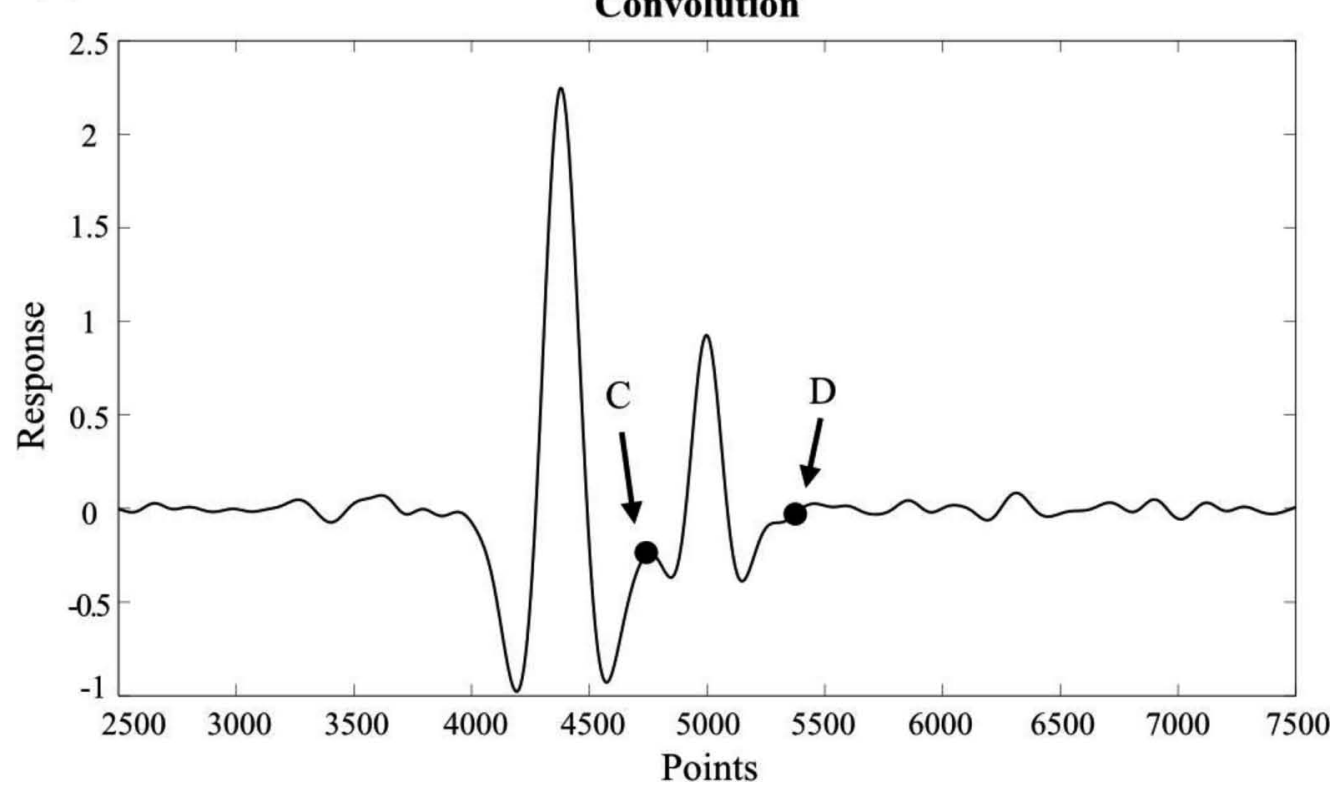

Fig. 3 (a) Segment of the original static LS chromatogram in Fig. 2 between the retention volume, 2.68 and $16.0 \mathrm{~mL}$, is re-plotted by replacing the retention volume in the $x$-axis as a sequence of data points. The estimated end points of the original pullulan $5 \mathrm{k}$ peak, are marked as A and B, respectively. (b) The zoom-in chromatogram in the central part of the filtered chromatogram resulting from convolving the original static LS chromatogram with the second-derivative trace in the imbedded graph in the upper frame. The left and right projected points of both edges (A and B in the upper frame) are marked as $\mathrm{C}$ and $\mathrm{D}$ in the filtered chromatogram.

filtered chromatogram. Points C and D are the 4758-th and 5402-th point, respectively.

In addition, when the M-th data point on the original chromatogram is convolved with the $\mathrm{N}$-th data point on the reference profile, the convolution result will be projected to the number $\mathrm{M}+\mathrm{N}-1$ data point on the filtered chromatogram. Using the location of interested points in Fig. $3 \mathrm{~b}$, where $\mathrm{M}+\mathrm{N}-1$ is specified as 4758 or 5402 with respect to the marked points $\mathrm{C}$ and $\mathrm{D}$, respectively, when $\mathrm{N}$ is specified as 2373 or 2628 , respectively, the values of $\mathrm{M}$ are found as 2386 and 2775 to locate the edge points of the original pullulan $5 \mathrm{k}$ peak, marked as A and B, respectively, in the original chromatogram (Fig. 3a). 
Table 1 Estimated molecular-weight values of pullulan standards

Standard of pullulan $5 \mathrm{k}$ (Molecular weight/6438 Da)

\begin{tabular}{cccc}
\hline & Estimated molecular weight/Da & Accuracy, $\%$ & Precision, $\%$ \\
\hline Using original chromatographic peak range & $7036 \pm 1313$ & 9.30 & 18.66 \\
Using filtered chromatographic peak range & $6352 \pm 1130$ & -1.32 & 17.80 \\
\hline Standard of pullulan 400 k (Molecular weight/383250 Da) & & & \\
\hline & Estimated molecular weight/Da & Accuracy, $\%$ & Precision, $\%$ \\
\hline Using original chromatographic peak range & $386470 \pm 28798$ & 0.84 & 7.45 \\
Using filtered chromatographic peak range & $377420 \pm 24373$ & -1.52 & 6.46 \\
\hline
\end{tabular}

Having found more precise end points of the SEC chromatographic peak acquired with the static LS detector, we are able to choose a more appropriate peak width range to calculate the $M_{\mathrm{W}}$ values using the OmniSEC software pack. Using eleven replicate sample injections the average results of the determined $M_{\mathrm{W}}$ values of pullulan $5 \mathrm{k}$ is $6352 \mathrm{Da}$, with the improved accuracy of $-1.3 \%$. This improvement of the $M_{\mathrm{W}}$ determination accuracy is significantly superior to the determination accuracy of the $M_{\mathrm{W}}$ value $(9.3 \%)$ without using the filtered peak width range.

Similarly, when the static LS chromatogram in Fig. 3a is convolved with the second-derivative profile of one Gaussian peak of that the center and standard deviation are estimated with the RI peaks of the pullulan $400 \mathrm{k}$, the end points of the convolved pullulan $400 \mathrm{k}$ peak are found to estimate the edge points of the original LS chromatographic peak. Also, when the more precise end points of the pullulan $400 \mathrm{k}$ peak on the original static LS chromatogram are found to define a more accurate peak width range to calculate the $M_{\mathrm{W}}$ values, using eleven replicate sample injections the average results of the determined $M_{\mathrm{W}}$ value of pullulan $400 \mathrm{k}$ is $377420 \mathrm{Da}$, of which the accuracy is $-1.5 \%$, similar to the accuracy $(0.8 \%)$ without using any filtering procedures.

Table 1 shows there are no significant differences between the determined precisions of the $M_{\mathrm{W}}$ values of pullulan $400 \mathrm{k}$, both around $7 \%$ using these two different methods. When the $M_{\mathrm{W}}$ values of pullulan $5 \mathrm{k}$ are determined with and without using a second-derivative filter to estimate the peak width range, the precisions are 18 and $19 \%$, respectively. The difference between these two precision values is also minor when somewhat excess numbers of replicate sample injections are used $(n=11)$. When the numbers of replicates are of typical size $(n=5)$, the determination precision difference between with and without using second-derivative filtering procedures becomes obvious. Using a second-derivative filter, the $M_{\mathrm{W}}$ determination precision of pullulan $400 \mathrm{k}$ and pullulan $5 \mathrm{k}$ have been improved from 9.8 to $4.7 \%$ and from 23.5 to $17.3 \%$, respectively. On the other hand, as discussed beforehand, the accuracy of using a second-derivative filter to determine the $M_{\mathrm{W}}$ value becomes obviously superior to the accuracy without using the filter, when the original static LS chromatographic signal peaks are too noisy to reliably estimate the peak width ranges. When a second-derivative filter is used, the $M_{\mathrm{W}}$ determination accuracy of pullulan $5 \mathrm{k}$ is $-1.3 \%$, significantly superior to the accuracy without using the filter, $9.3 \%$. When the $M_{\mathrm{W}}$ values of pullulan $400 \mathrm{k}$ are determined, the accuracy are both less than $2 \%$ with and without using second-derivative filters.

The second-derivative filter is one type of band-pass filter. This filter is highly efficient to reduce a variety of noises, such as spike noises and shifting baselines, which contain significant amounts low-frequency components, ${ }^{11}$ inherent in chromatographic signal peaks acquired with a static LS detector. Therefore, the chromatographic peak signals are properly tailored with the filter and the precise peak width ranges are more easily determined.

\section{Conclusions}

In this paper, using a second-derivative filtering method to smooth and correct static LS chromatographic peaks prior to estimating the peak width ranges is proved to be a useful technique to obtain an improved $M_{\mathrm{W}}$ determination accuracy for polymers such as pullulan $5 \mathrm{k}$, of which the original signal peak is noisy.

\section{Acknowledgements}

The authors are thankful for financial support from the Ministry of Science and Technology, Taiwan (100-2113-M-194-001-MY3, and 102-2627-M-194-001).

\section{References}

1. A. M. Striegel, "Multiple Detection in Size Exclusion Chromatography" (Vol. 893, ACS Symposium Series), 2004, American Chemical Society, Washington, D.C.

2. B. H. Zimm, J. Chem. Phys., 1948, 16, 1093.

3. R. Strelitzki and W. F. Reed, J. Appl. Polym. Sci., 1999, 73, 2359.

4. I. Suarez and B. Coto, Eur. Polym. J., 2011, 47, 2331.

5. R. Chen, N. Ilasi, and S. S. Sekulic, J. Pharm. Biomed. Anal., 2011, 56, 743.

6. M. Shakun, H. Maier, T. Heinze, P. Kilz, and W. Radke, Carbohydr. Polym., 2013, 95, 550.

7. A. Oliva, M. Llabrés, and J. B. Fariña, J. Chromatogr. A, 2014, 1353, 89.

8. C. D. Brown, L. Vega-Montoto, and P. D. Wentzell, Appl. Spectrosc., 2000, 54, 1055.

9. J. Wang, Q. Qiu, and W. Liu, Guangpuxue Yu Guangpu Fenxi, 2007, 27, 895.

10. R. Danielsson, D. Bylund, and K. E. Markides, Anal. Chim. Acta, 2002, 454, 167.

11. S. C. Wang, C. J. Lin, S. M. Chiang, and S. N. Yu, Anal. Chem., 2008, 80, 2097. 